\title{
Robot-assisted laparoscopic pyeloplasty: a review of minimally invasive treatment options for ureteropelvic junction obstruction
}

\author{
Vipul R. Patel · Nilesh N. Patil · Geoff Coughlin • \\ Pankaj P. Dangle $\cdot$ Kenneth Palmer
}

Received: 11 August 2007 / Accepted: 19 August 2007 / Published online: 8 January 2008

C) Springer London 2008

\begin{abstract}
Our aim was to examine the current status of minimally invasive approaches to ureteropelvic junction (UPJ) obstruction and compare it to the gold standard of open pyeloplasty. A review of the literature was conducted using PubMed and Medline databases for UPJ obstruction. Open pyeloplasty achieves good results, in the range of 90-100\%. Laparoscopic pyeloplasty results are as good as those of open surgery. However, the goal of laparoscopic pyeloplasty as a means of providing minimally invasive surgery to a larger number of patients has not been achieved. The reason for this is the difficulty faced by most urologists in acquiring the technical skills to perform a laparoscopic pyeloplasty. In reports of robot-assisted pyeloplasty, results in the range of $88-97 \%$ appear to be achieved. Robotic technology has the potential to make minimally invasive pyeloplasty an easier skill to acquire for a larger number of urologists. Long-term data are still required to determine its efficacy.
\end{abstract}

Keywords Laparoscopic - Robotic ·

Ureteropelvic junction obstruction

\section{Introduction}

Ureteropelvic junction (UPJ) obstruction is a functionally significant impedance of urine flow from the renal pelvis into the ureter. Congenital UPJ obstruction is one of the

V. R. Patel $(\varangle) \cdot$ N. N. Patil · G. Coughlin · P. P. Dangle ·

K. Palmer

Ohio State University, Columbus, Ohio, USA

e-mail: vipul.patel.md@ flhosp.org most common congenital abnormalities of the ureter. The etiology may be congenital or acquired. Here, the obstruction is typically caused by an aperistaltic segment of the collecting system. The role of lower pole crossing vessels remains controversial. Acquired causes are less common and include stone disease, postoperative or inflammatory strictures, benign and malignant neoplasms and extrinsic compression.

Traditionally the treatment of UPJ obstruction has been surgical, the gold standard of which has been the open pyeloplasty. There are many surgical approaches that have been used over the years, such as the anterior retroperitoneal technique and the posterior lumbotomy method.

In 1949 Anderson and Hynes described the open dismembered pyeloplasty [1]. This technique has been used as the template for open pyeloplasty. The method allows complete excision of an anatomically abnormal UPJ and the reduction of a redundant renal pelvis. With a success rate in excess of $90 \%$ [2], open pyeloplasty is seen as the gold standard treatment for UPJ obstruction. However, pain and prolonged convalescence have been its major drawbacks.

Over the past two decades, minimally invasive surgery has been utilized with increasing frequency in the field of urological surgery. This is true for the surgical management of UPJ obstruction [3, 4]. Minimally invasive options aim to offer less morbidity and shorter convalescence periods than open surgery. Minimally invasive operations for UPJ obstruction include endoscopic endopyelotomy techniques, laparoscopic pyeloplasty and robot-assisted pyeloplasty.

Introduction of robotic technology into modern day surgery has the potential to offer significant advantages. The wristed instruments, $\times 10$ magnifications, tremor filtering, scaling of movements and three-dimensional view allow the urologist to perform the intricate dissection and anastomosis of the collecting system with high precision. 
This paper reviews the treatment options for UPJ obstruction, primarily focusing on the current status of robot-assisted pyeloplasty

\section{Methods}

We performed a systematic search of PubMed and Medline databases for UPJ obstruction. Papers were examined for demographic data, intraoperative parameters and postoperative outcomes.

\section{Endopyelotomy}

Endopyelotomy involves making a full thickness incision through the obstructing ureteropelvic junction and allowing the urothelium and ureteric smooth muscle to regenerate around a ureteric stent. The approach to the UPJ can be antegrade or retrograde. The endopyelotomy can be made under direct vision or fluoroscopic guidance with either diathermy or laser. Success rates for endopyelotomy vary from $65 \%$ to $90 \%$ [5, 6]. While endopyelotomy is, perhaps, the most minimally invasive treatment approach, it does have limitations. The success rate is less than that of the gold standard open pyeloplasty. Endopyelotomy cannot establish dependant drainage of the UPJ, nor can it correct for anterior crossing vessels. Significant intraoperative and postoperative bleeding is one of the major concerns of endopyelotomy, with an incidence of 8-9\% [7]. Endopyelotomy is thought to be an inappropriate treatment for UPJ obstruction associated with significant hydronephrosis, poor renal function or long UPJ stricture [8].

Pyeloplasty

Pyeloplasty can be performed by an open, laparoscopic or robotic technique. Table 1 compares the approaches, operating times, follow-up periods and successes of these various techniques.

\section{Open pyeloplasty}

Open pyeloplasty is the gold standard treatment for correction of UPJ obstruction. Open pyeloplasty can be performed via an anterior or posterior approach. Various surgical techniques have been described to correct the UPJ obstruction, including dismembered pyeloplasty, V-Y pyeloplasty [9], Heinke-Miculikz pyeloplasty [10] and spiral flaps [11]. Reported success rates for open pyeloplasty are in excess of $90 \%$ [2]. The main drawbacks of open pyeloplasty are the long recovery time and the morbidity of the flank incision.

\section{Laparoscopic pyeloplasty}

Laparoscopic pyeloplasty aims to adhere to the surgical principles of open pyeloplasty. It is a minimally invasive surgical option that provides the desirable aspects of open surgery, including the precise mucosal approximation, excision of redundant renal pelvis tissue, and anterior transposition of the UPJ for lower pole crossing vessels. The operation can be performed through a retroperitoneal or a transperitoneal approach. The first laparoscopic pyeloplasty was reported by Schuessler et al. [12]. Since then various series have quoted success rates of $85-100 \%$, closely approximating that of open pyeloplasty [13].

The initial large series of 100 patients reported in 2002 by Jarrett et al. [14] reviewed their experience with laparoscopic pyeloplasty. The repair was carried out in 83 patients for primary UPJ obstruction and in 17 for secondary obstruction. Average operating time was $4.2 \mathrm{~h}$, which decreased with surgeon experience. Average blood loss was $181 \mathrm{ml}$, and hospital stay was 3.3 days. Radiographic success was defined as improvement of hydronephrosis, with a patent ureteropelvic junction shown on intravenous pyelogram (IVP), or improved drainage on diuresis renography. With a mean clinical and radiographic follow-up period of 2.7 years and 2.2 years, respectively, $96 \%$ of the patients were free of obstruction on follow-up imaging. The authors
Table 1 Characteristics and outcomes of open, laparoscopic and robot-assisted pyeloplasty (Lap laparoscopic, Retro retroperitoneal, Trans transperitoneal, $N M$ not mentioned)

\begin{tabular}{lllllll}
\hline Reference & Method & Number & Approach & $\begin{array}{l}\text { Operating } \\
\text { time (min) }\end{array}$ & $\begin{array}{l}\text { Follow-up } \\
\text { period (months) }\end{array}$ & $\begin{array}{l}\text { Success } \\
\text { rate (\%) }\end{array}$ \\
\hline Lee et al. [23] & Open & 33 & Retro & 181 & $20(1-57)$ & 100 \\
Gogus et al. [24] & Open & 180 & Retro & NM & 12 & 91 \\
Moon et al. [15] & Lap & 170 & Retro & 170 & $15(3-72)$ & 97 \\
Inagaki et al. [25] & Lap & 147 & Trans & 140 & $24(3-84)$ & 98 \\
$\begin{array}{l}\text { Schwentner and } \\
\quad \text { Pelzer [20] }\end{array}$ & Robotic & 92 & Trans & 108 & $39(3-73)$ & 97 \\
$\begin{array}{l}\text { Patel [19] } \\
\text { Palese et al. [26] }\end{array}$ & Robotic & 50 & Trans & 122 & 11 & 96 \\
Mendez-Torres & Robotic & 38 & Trans & 225 & 12 & 94 \\
$\quad$ et al. [27] & & Trans & 300 & 8 & 88 \\
\hline
\end{tabular}


opined that the disadvantages of the procedure remain the long operative times, and the need for special skills for intracorporeal tying and suturing can increase hospital costs.

The largest series of laparoscopic pyeloplasty has been reported by Moon et al. [15]. They reported their data on 170 consecutive laparoscopic pyeloplasties (156 for primary and 14 for secondary UPJ obstruction). The average operating time was $140 \mathrm{~min}$. Median hospital stay was for 3 days. There were 12 complications reported. With a mean follow-up period of 15 months, the success rate was $96.2 \%$. Success was defined by the presence of each of three criteria: a decreasing renographic excretion curve or proven anastomotic patency, improved differential renal function and the absence of pain.

Despite the success of this minimally invasive approach, laparoscopic pyeloplasty is largely being performed only by expert laparoscopists. The technical difficulty in preparing and performing the anastomosis has limited the enthusiasm for this operative approach.

\section{Robotic pyeloplasty}

The da Vinci Surgical System (Intuitive Surgical, Sunnyvale, CA, USA) offers several advantages for laparoscopic pyeloplasty. It consists of a remote computer console from which the surgeon can observe the actions of the instrumentation with a magnified $(\times 10)$ three-dimensional view. The robot is attached directly to the laparoscopic trocars through which the instruments are inserted. These arms are controlled via the surgical joysticks into which the surgeon inserts his/her fingers. The joysticks allow free movement that is translated into seven degrees of freedom at the robotic instrument tip. The tips of these instruments are small and able to articulate freely, with scaling and tremor filtration.

Robotic pyeloplasty was first performed by Sung and Gill [16] in 1995 on a porcine model. Their study compared the pyeloureteric anastomosis time and tightness of the anastomosis between intracorporeal suturing and robot-assisted suturing. The robot-assisted suturing had increased anastomosis time, but the tightness of the anastomosis was equal in both groups. Later, this same group compared the Zeus and da Vinci systems. They were able to perform the anastomosis faster and secure it with more bites using the da Vinci system. The comparison between the two systems revealed that the da Vinci was more technically intuitive to use and thereby had the potential to decrease the learning curve.

\section{Operative technique}

The robotic pyeloplasty can be performed either retroperitoneally or transperitoneally. We have previously described our transperitoneal operative approach in detail [17]. Initially, an ascending ureteropyelogram is performed and a ureteric stent is placed (Fig. 1). The patient is positioned in a modified flank position with a $30^{\circ}$ incline. The operating table and the da Vinci platform are positioned at either a perpendicular or a $45^{\circ}$ angle relative to one another, depending upon trocar placement (Fig. 2). Figure 3 illustrates the standard port placement for a right-sided transperitoneal pyeloplasty. A hybrid procedure with laparoscopic exposure of the UPJ followed by robotic assistance to prepare and perform the anastomosis is our preferred technique. The bowel is mobilized medially, laparoscopically. Gerota's fascia is opened, and the ureter is identified (Fig. 4). The dissection proceeds superiorly to display the UPJ and determine the presence or absence of crossing vessels (Fig. 5). Care is taken not to devascularize the ureter with extensive dissection. The UPJ is assessed to determine the type of repair which is most suitable. For a dismembered pyeloplasty, the UPJ is cut, spatulated, and, if necessary, anteriorly transposed (Fig. 6). During spatulation the advantages of wristed instruments are significant, as they allow a more precise linear incision. A running anastomosis is performed over a $6 \mathrm{Fr}$ ureteric stent using two $5 \mathrm{in}$. running 4-0 monocryl sutures on RB-1 needles (Fig. 7).

\section{Clinical outcomes}

The initial series was reported by Gettman et al. [18], who performed the classical Anderson and Hynes dismembered pyeloplasty in nine patients, using the da Vinci robot. They

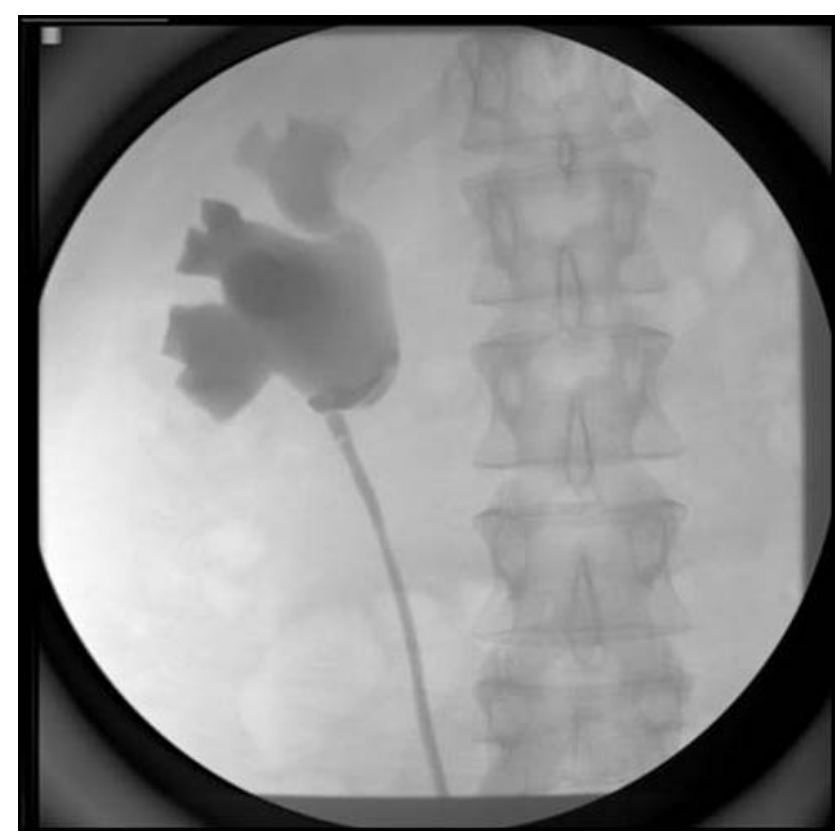

Fig. 1 Ascending ureteropyelogram is performed and a ureteric stent is placed 


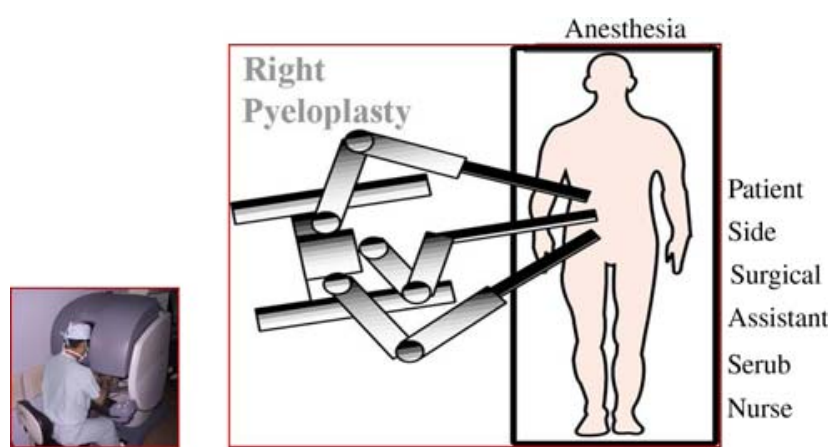

Fig. 2 Trocar placement

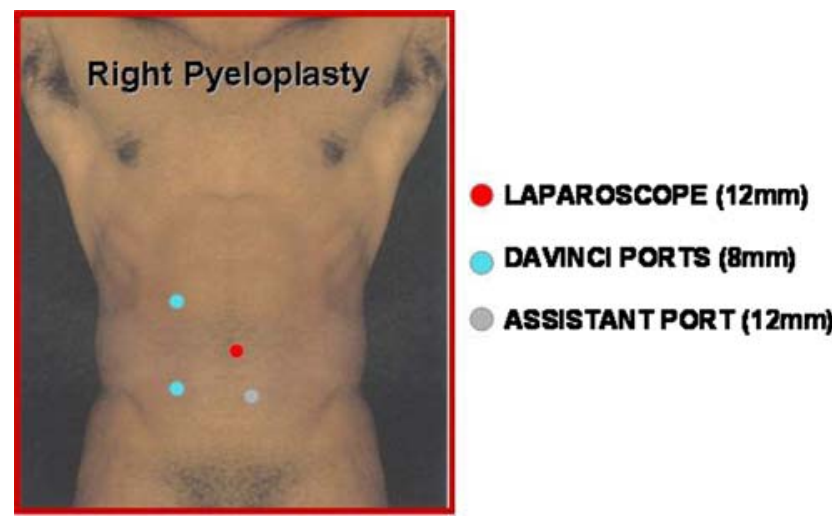

Fig. 3 Trocar placement in transperitoneal pyeloplasty

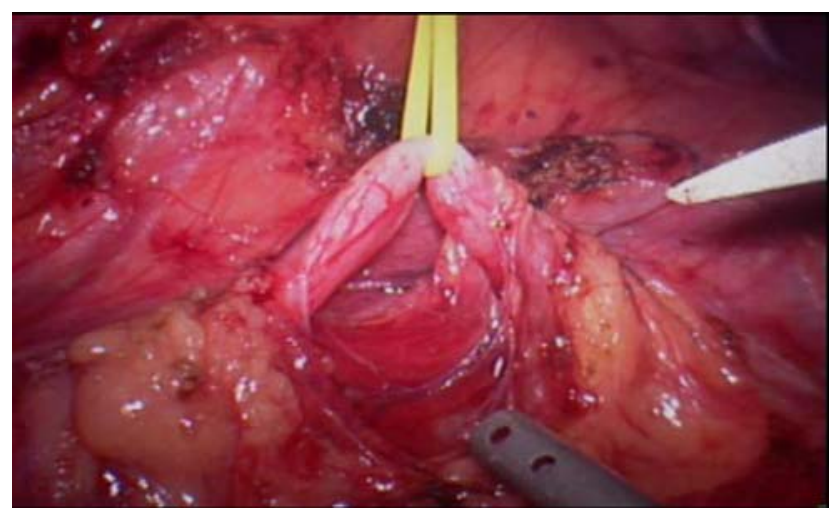

Fig. 4 Ureter identified after the opening of Gerota's fascia

used three robotic ports and one port for the assistant. Mean operative time was $138.8 \mathrm{~min}$. Average blood loss was $<50 \mathrm{ml}$, and the mean hospital stay was 4.7 days. At a mean follow-up time of 4.1 months, all procedures were successful on the basis of subjective and radiographic data.

We reported on 50 [19] patients who had undergone robot-assisted dismembered pyeloplasty. The average operative time was $122 \mathrm{~min}(60-330 \mathrm{~min})$. The average hospital stay was 1.1 day. The average blood loss was $40 \mathrm{ml}$. The average follow-up period was 11 months. Patients were followed up by renogram and clinically. Ninety-six percent

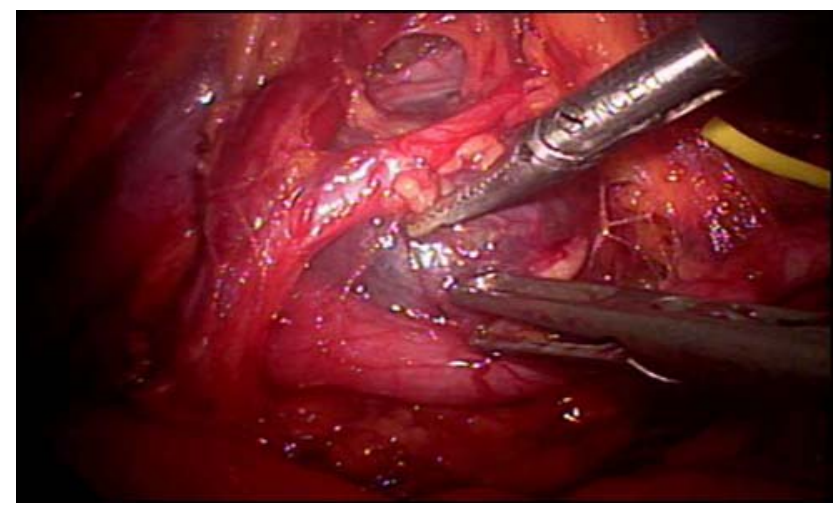

Fig. 5 Crossing vessel

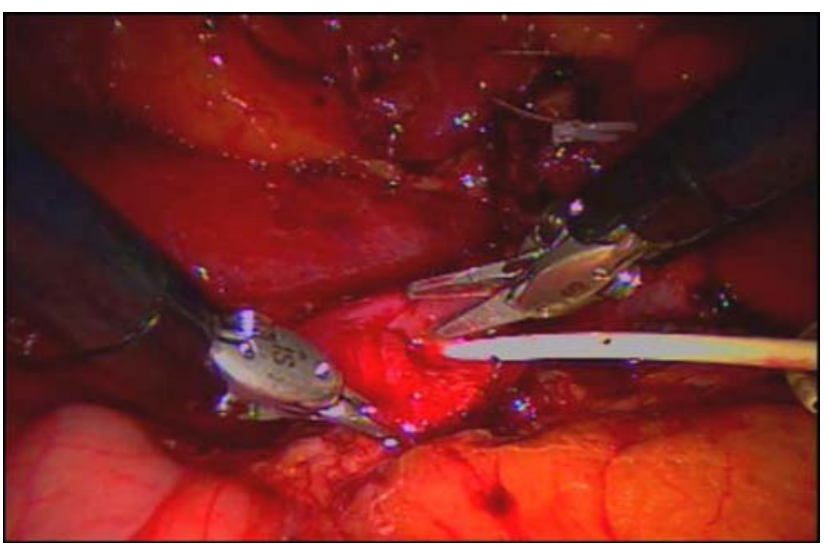

Fig. 6 Spatulated ureter

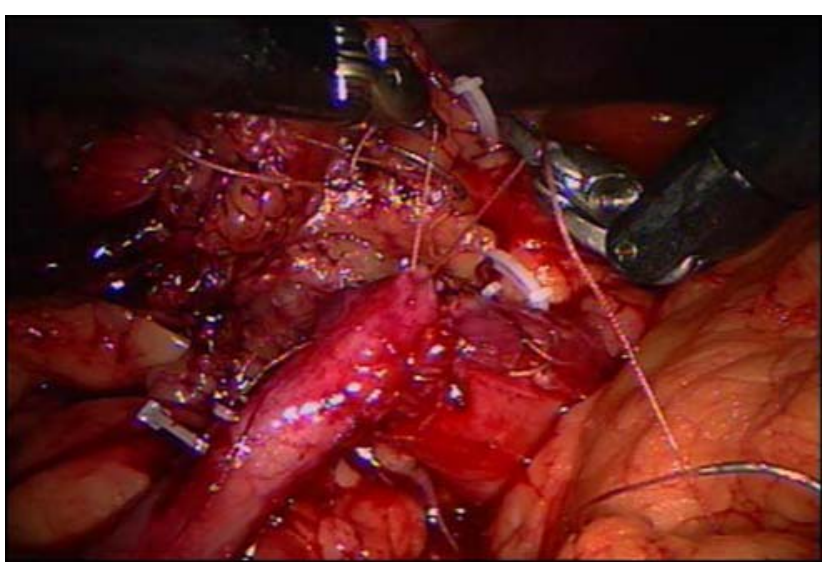

Fig. 7 Continuous anastomosis

showed improvement in their radiological, functional and clinical parameters.

Schwentner and Pelzer [20] recently reported the largest experience of the robot-assisted pyeloplasty. Ninety-two patients underwent robot-assisted dismembered pyeloplasty for UPJ obstruction. The mean operative duration, including the setup of the robot, was $108.34(72-215)$ min. The mean hospital stay was 4.5 days. They reported a mean follow-up time of 39.1 months and a success rate of $97 \%$. Suc- 
cess was measured on the basis of an intravenous urogram (IVU), mercapto-acetyl tri-glycine (MAG3) scintigraphy (at 3 months) and resolution of subjective complaints.

Weise and Winfield [21] reported their experience with 31 patients who underwent robotic pyeloplasties. Operative time, including cystoscopy, was $271 \mathrm{~min}$ in this group. The estimated median blood loss was $<100 \mathrm{ml}$. The median console time was $76 \mathrm{~min}$ (range 54-124 min) and consisted of preparation and completion of the anastomosis. There were no conversions to open surgery. The mean follow-up period was 10 months (1-31 months). The success rate, defined as no evidence of persistent obstruction on renogram, no loss of function and no postoperative pain, was $97 \%$.

Atug et al. [22] reported their experience with robotic laparoscopic surgery for UPJ obstruction with concomitant management of renal calculi in eight patients. The mean operative time was $275 \mathrm{~min}$ (range 180-345 $\mathrm{min}$ ). The console time was $181 \mathrm{~min}$ (125-222 min). The mean blood loss was $48.6 \mathrm{ml}(10-100 \mathrm{ml})$. The mean hospital stay was 1.1 day (1-2 days). Before completing the UPJ obstruction repair one robotic arm was undocked to allow a flexible nephroscope to be passed into the renal pelvis for stone removal. There were no open conversions.

Robot-assisted retroperitoneal dismembered pyeloplasty was described for the first time by Hegarty et al. (N. J. Hegarty, J. H. Kaouk, S. J. Parekattil, Robotic assisted retroperitoneal dismembered pyeloplasty-technique and outcomes at 1 year. Proceedings of the 24th World Congress of Endourology, 17-20 August 2006; Cleveland, OH, USA, unpublished data) in ten adult patients. Access to the retroperitoneum was achieved by a balloon dissector. The mean operative time was $157 \mathrm{~min}$, with a mean blood loss of $50 \mathrm{ml}$ and a mean hospital stay of $48 \mathrm{~h}$. All cases were completed using the robot. The authors reported a successful outcome, clinically and radiologically, in all patients after a mean follow-up time of 12 months.

\section{Pediatric robot-assisted pyeloplasty}

Advances in robotic technology have allowed complex reconstructive surgeries even in the pediatric population. Lee et al. [23] have presented their series of comparisons between open pyeloplasty (33 patients) and robot-assisted pyeloplasty (33 patients). In the robot-assisted pyeloplasty group the mean age was 7.9 years and mean operative time was $219 \mathrm{~min}$, with a mean estimated blood loss of $3 \mathrm{ml}$. There was one complication of bleeding, in which a crossing vessel was not recognized. This was the only patient who had undergone a retroperitoneal robot-assisted pyeloplasty. The patient subsequently required percutaneous drainage and transperitoneal pyeloplasty. Mean operative time was $181 \mathrm{~min}$ for open pyeloplasty versus $219 \mathrm{~min}$ for robot-assisted pyeloplasty. The length of stay for those undergoing robot-assisted pyeloplasty was 2.3 days versus 3.5 days for those undergoing open pyeloplasty. Total narcotics requirements were significantly fewer in the robotassisted pyeloplasty group. Although operative times were increased compared with those of open pyeloplasty, Robotassisted pyeloplasty (RALP) demonstrated decreased hospital stay and narcotics use. The authors concluded that robot-assisted pyeloplasty is a safe and efficacious procedure in children and that increasing experience with RALP decreased operative time approximating that of the open experience.

\section{Discussion}

In the recent past there has been a number of studies which have highlighted the advantages of laparoscopic pyeloplasty $[14,15]$. The success rates reported in most series have been comparable with the gold standard of open pyeloplasty. However, the special skills of intracorporeal suturing and tying have not percolated to a large number of urologists. Thus, the utility of laparoscopic pyeloplasty has been limited to the "experts" in laparoscopy.

Robotic technology with its inherent advantages, besides being minimally invasive, of magnified image, threedimensional view, tremor filtration, motion scaling and wristed instruments has leveled the playing field and made it available to a large number of patients. The robot was initially looked upon as a tool to help the surgeon make a transition from open to laparoscopic surgery. However, its use in various complex urological reconstructive procedures has shown that the robot has broader applications than the standard laparoscopic pyeloplasty. It has brought the technical skills of minimally invasive surgery within the realm of a greater number of urologists.

\section{Conclusion}

The early outcome data in robot-assisted pyeloplasty is encouraging. Further follow-up and long-term data are required to determine its efficacy in the long run. As more centers and more urologists train to perform robot-assisted pyeloplasty, it has the potential to become the minimally invasive treatment of choice.

\section{References}

1. Anderson JC, Hynes W (1949) Retrocaval ureter: a case diagnosed preoperatively and treated successfully by a plastic operation. Br J Urol 21:209 
2. O'Reilly PH, Brooman PJC, Mak S et al (2001) The long-term results of Anderson-Hynes pyeloplasty. BJU Int 87:287-289

3. Shah K, Louie M, Thaly R, Patel VR (2007) Robot assisted laparoscopic pyeloplasty: a review of the current status. Int J Med Robot 3:35-40

4. Janetschek G, Peschel R, Franscher F (2000) Laparoscopic pyeloplasty. Urol Clin North Am 27:695-704

5. Knudsen BE, Cook AJ, Watterson JD, Beiko DT (2004) Percutaneous antegrade endopyelotomy: long-term results from one institution. Urology 63:230-234

6. Kletscher BA, Segura JW, Le Roy AJ et al (1995) Percutaneous antegrade endopyelotomy: review of 50 consecutive cases. J Urol 153:701-703

7. Aslan P, Preminger GM (1998) Retrograde balloon cautery incision of ureteropelvic junction obstruction. Urol Clin North Am 25:295-304

8. Motola JA, Fried R, Badlani GH, Smith AD (1993) Failed endopyelotomy: implications for future surgery on the ureteropelvic junction. J Urol 150:821-823

9. Foley FB (1937) New plastic operation for stricture at the ureteropelvic junction. J Urol 38:643

10. Fenzer C (1984) Operation for the relief of valve formation and stricture of the ureter in hydro or pyonephrosis. JAMA 22:335

11. Scardino PL, Prince CL (1953) Vertical flap ureteropelvioplasty: preliminary report. South Med J 46:325

12. Schuessler WW, Grune MT, Tecuanhuey LV, Preminger GM (1993) Laparoscopic dismembered pyeloplasty. J Urol 150:1795-1799

13. Munver R, Sosa R, Joseph J Del Pizzo (2004) Laparoscopic pyeloplasty: history, evolution, and future. J Endourol 18:748-755

14. Jarrett T, Chan D, Charambura T (2002) Laparoscopic pyeloplasty: the first 100 cases. J Urol 167:1253-1256

15. Moon DA, El-Shazly MA, Chang CM, Gianduzzo TR, Eden CG (2006) Laparoscopic pyeloplasty: evolution of a new gold standard. Urology 5:932-936
16. Sung G, Gill I (2001) Robotic laparoscopic surgery: a comparison of the da Vinci and Zeus systems. Urology 58:893-898

17. Louie M, Carey R, Leveillee R, Patel VR (2007) Robotic pyeloplasty. In: Patel VR (ed) Robotic urologic surgery. Springer, London, pp 152-160

18. Gettman M, Neururer R, Bartsch G (2002) Anderson-Hynes dismembered pyeloplasty performed using the da Vinci robotic system. Urology 60:509-513

19. Patel VR (2005) Robotic assisted laparoscopic dismembered pyeloplasty. Urology 66:45-49

20. Schwentner C, Pelzer A, Neururer R et al (2007) Robotic Anderson-Hynes pyeloplasty: 5-year experience of one centre. BJU Int 100:880-885. doi: 10.1111/j.1464-410X.2007.07032.x

21. Weise E, Winfield H (2006) Robotic computer-assisted pyeloplasty versus conventional laparoscopic pyeloplasty. J Endourol 20:813-819

22. Atug F, Castle E, Scott B, Thomas R (2005) Concomitant management of renal calculi and pelvi-ureteric junction obstruction with robotic laparoscopic surgery. BJU Int 96:1365-1368

23. Lee R, Retik A, Boreer J (2006) Pediatric robot assisted laparoscopic dismembered pyeloplasty: comparison with a cohort of open surgery. J Urol 175:683-687

24. Gogus C, Karamursel T, Tokatli Z et al (2004) Long-term results of Anderson-Hynes in 180 adults in the era of endourologic procedures. Urol Int 73:11-14

25. Inagaki T, Kavoussi LR, Jarrett TW (2005) Laparoscopic pyeloplasty: current status. BJU Int 95:102-105

26. Palese MA, Stifelman MD, Munver R (2005) Robot-assisted laparoscopic dismembered pyeloplasty: a combined experience. J Endourol 19:382-386

27. Mendez-Torres F, Woods M, Thomas R (2005) Technical modifications for robot-assisted laparoscopic pyeloplasty. J Endourol 19:393-396 